\title{
Dynamics and morphology of solid electrolyte interphase (SEI)
}

\author{
Fabian Single $^{\text {ab }}{ }^{\text {Birger Horstmann }}{ }^{* a b}$ and Arnulf Latz $^{\text {abc }}$ \\ *Corresponding author: E-mail: birger.horstmann@dlr.de \\ ${ }^{a}$ German Aerospace Center (DLR), Institute of Engineering Thermodynamics, Pfaffenwaldring 38-40, 70569 \\ Stuttgart, Germany \\ ${ }^{b}$ Helmholtz Institute Ulm (HIU), Helmholtzstraße 11, 89081 Ulm, Germany \\ ${ }^{c}$ Ulm University, Institute of Electrochemistry, Albert-Einstein-Allee 47, 89069 Ulm, Germany
}

Phys. Chem. Chem. Phys., 2016,18, 17810-17814

DOI: $10.1039 / C 6 C P 02816 K$

Received 27 Apr 2016, Accepted 13 Jun 2016

First published online 13 Jun 2016

We develop a novel theory for the continuous electrochemical formation of porous films to study the solid electrolyte interphase (SEI) on lithium ion battery anodes. Existing SEI studies model a homogeneous morphology and a single relevant transport mechanism. Our approach, in contrast, is based on two transport mechanisms and enables us to track SEI porosity in a spatially resolved way. SEI thickness evolution agrees with existing studies and is validated with experiments. This consistent approach is unprecedented in SEI modeling. We predict a non-zero SEI porosity and the dependence of morphology on transport properties. Additionally, we capture dual-layer chemistry and morphology. Analytic expressions which describe the parameter dependence of all key properties are derived and discussed. 